\title{
Pascal - la religion comme expérience de la contingence et de l'altérité
}

\author{
Jimmy Sudário Cabral* \\ Peut-être le bonheur adviendra-t-il au détour d'un boulevard, \\ dans la pleine présence d'une femme, dans la joie \\ si intensément réelle d'une rencontre aussi improbable que \\ celle de Dieu en personne (Gérard Bensussan, 2019, 47)
}

\section{Résumé}

L'article analyse l'interprétation de Pascal du concept de religion et la configuration anarchique du christianisme présente dans son œuvre Pensées. À la lumière des concepts centraux de l'œuvre pascalienne, tels que l'équivocité, la disproportion et la contingence, et de son dialogue avec la pensée de Descartes et Montaigne, l'article présente le rejet pascalien des concepts de nature, intériorité et principe, fondamentaux dans l'élaboration de certain vocabulaire philosophique et mystique. Nous soutenons que le dialogue de Pascal avec le scepticisme et le rationalisme offrait une propédeutique à sa pensée religieuse et articulait une grammaire philosophique religieuse résistante à a) un vocabulaire mystique aux racines néoplatoniques b) une philosophie panthéiste de la nature et c) au geste ontothéologique qui donna naissance à ce que Pascal appela le dieu des philosophes.

Mots-clés: Pascal, religion, christianisme, philosophie, contingence

\section{Pascal: a religião como experiência da contingência e da alteridade}

\section{Resumo}

$\mathrm{O}$ artigo analisa a interpretação de Pascal do conceito de religião e a configuração anárquica do cristianismo presente em sua obra Pensées. À luz de conceitos centrais na obra pascaliana, tais como equivocidade, desproporção e contingência, e do seu diálogo com o pensamento de Descartes e Montaigne, o artigo apresenta a rejeição pascaliana dos conceitos de natureza, interioridade e princípio, fundamentais na elaboração de certo vocabulário filosófico/teológico e místico. Argumentamos que o diálogo de Pascal com o ceticismo e o racionalismo ofereceu uma propedêutica ao seu pensamento religioso e

\footnotetext{
* Universidade Federal de Juiz de Fora. jimmy.sudario@gmail.com .
} 
articulou uma gramática filosófico religiosa resistente a a) um vocabulário místico com raízes neoplatônicas, b) uma filosofia panteísta da natureza e c) ao gesto ontoteológico que deu à luz ao que Pascal chamou de deus dos filósofos.

Palavras-chave: Pascal; religião; cristianismo; filosofia; contingência

\title{
Pascal: Religion as an Experience of Contingency and Otherness
}

\begin{abstract}
This paper analyzes Pascal's Pensées interpretation on religion and the anarchic configuration of Christianity. According to Pascal's work main concepts - such as equivocity, disproportion and contingency — and in his dialogue with Descartes and Montaigne thoughts, the paper presents the pascalienne rejection of the concepts of nature, interiority and principle, fundamental in the elaboration of a certain philosophical and mystical vocabulary. We argue that Pascal's dialogue with skepticism and rationalism offered a propaedeutic to his religious thought and articulated a religious philosophical grammar resistant to: a) a mystical vocabulary with Neoplatonic roots, b) a pantheistic philosophy of nature, and c) the ontotheological gesture that gave birth to what Pascal called the god of philosophers.
\end{abstract}

Keywords: Pascal; religion; christianity; philosophy; contingency

\section{Pascal: la religión como experiencia de contingencia y alteridad}

\section{Resumen}

El artículo analiza la interpretación de Pascal del concepto de religión y la configuración anárquica del cristianismo presente en su obra Pensées. A la luz de conceptos centrales en la obra pascual, como equívoco, desproporción y contingencia, y su diálogo con el pensamiento de Descartes y Montaigne, el artículo presenta el rechazo pascual de los conceptos de naturaleza, interioridad y principio, fundamentales en la elaboración de cierto vocabulario. filosófico / teológico y místico. Sostenemos que el diálogo de Pascal con el escepticismo y el racionalismo ofreció una propedéutica a su pensamiento religioso y articuló una gramática filosófica religiosa resistente a a) un vocabulario místico con raíces neoplatónicas, b) una filosofía panteísta de la naturaleza yc) el gesto ontoteológico que dio a luz a lo que Pascal llamó el dios.

Palabras clave: Pascal; religión; Cristiandad; filosofía; contingencia

\section{I. Équivocité}

Pascal fut, dans tout ce qu'il pensa et écrivit, un témoin de la profanation moderne de la cosmologie philosophique et religieuse des anciens et un observateur attentif d'une nature muette et de l'univers profané de la philosophie et de la science moderne. En élisant le scepticisme de 
Montaigne et le rationalisme de Descartes comme interlocuteurs, Pascal rejeta l'indifférence sceptique du premier et le rationalisme dogmatique du second, anticipant de deux siècles le nihilisme des modernes face à une nature vidée et un cosmos dépourvu de valeur morale (KOYRÉ, 1957, p.43). Sur le prisme philosophique des deux penseurs, Pascal fut un sceptique et un homme de sciences, et il sut mettre sub judice tout argument d'autorité non soumis à l'observation empirique. Les premières considérations de sa Préface sur le traité $d u$ vide nous offrent une image de sa place dans l'histoire de la pensée scientifique et de son rôle dans la querelle entre l'ancien et le moderne. Pour lui, «le respect que l'on porte à l'antiquité étant aujourd'hui à tel point, dans les matières où il doit avoir moins de force, que l'on se fait des oracles de toutes ses pensées et des mystères même de ses obscurités»(PASCAL, 1844, p.91). L'observation de Pascal sur le respect réservé à l'Antiquité reflète une position qui met en évidence l'absence de correspondance entre le rationalisme de Galilée et de Descartes et la tradition philosophico-théologique aristotélicienne et / ou néoplatonicienne. Dans son parcours philosophique et dans le cadre de l'élaboration des Pensées, qui est l'ouvrage fondamental pour comprendre sa trajectoire de penseur religieux, Pascal assimila les conséquences de la destruction de la cosmologie des anciens [comme nul autre en son temps] et attesta l'absence de tout principe théologique ou philosophique qui pourrait établir une expérience d'unité pour l'homme dans le monde.

Pascal devint très tôt indifférent aux modèles philosophiques de l'aristotélisme et résistant aux spéculations du néoplatonisme, établissant une critériologie qui cherchait à briser les limites de la pensée philosophique et scientifique à travers une confrontation avec le scepticisme de Montaigne et le rationalisme de Descartes. La critériologie de Pascal, appliquée dans les Pensées comme modèle d'apologétique religieuse du christianisme, part d'une renonciation à toute possibilité d'unité philosophique ou religieuse dans la nature et suppose la disproportion et la contingence absolue de l'expérience de l'homme dans le monde. Le scepticisme et le rationalisme modernes furent ainsi présentés comme une sorte de propédeutique à la pensée religieuse et supposèrent l'impossibilité de toute retraite vers un mysticisme aux racines néoplatoniciennes, vers une philosophie panthéiste de la nature ou vers une ontothéologie qui a donné naissance à l'idole métaphysique que Pascal appelait le dieu des philosophes. La forme fragmentée des Pensées, opposée à toute unité, peut être lue comme une combinaison 
entre l'atomisme scientifique de l'empirisme rationaliste et le geste essayiste de Montaigne ${ }^{1}$, et on y trouve l'articulation d'une grammaire religieuse résistante à tout idéalisme philosophique ou théologique : «la théologie est une science, mais en même temps combien est-ce de sciences ? Un homme est un suppôt, mais si on l'anatomise que sera-ce? la tête, le coeur, l'estomac, les veines, chaque veine, chaque portion de veine, le sang, chaque humeur de sang».(PASCAL, 1952, fr. 65).

La grammaire religieuse qui articule les Pensées admet l'insignifiance d'une science théologique dans la mesure où elle présuppose que «s'il y a un Dieu il est infiniment incompréhensible, puisque n'ayant ni parties ni bornes, il n'a nul rapport à nous. Nous sommes donc incapables de connaître ni ce qu'il est, ni s'il est. Cela étant qui osera entreprendre de résoudre cette question? Ce n'est pas nous qui n'avons aucun rapport à luì. (Frag. 418). L'argument de Pascal, qui part d'une critériologie qui reconnaît les limites des lumières naturelles, relie ces limites empiriques à la nature apophatique de la religion chrétienne et à l'idée qui est au cœur de son interprétation du christianisme: Dieu est un Dieu absconditus. Dans l'articulation de son herméneutique de la tradition judéo-chrétienne, Pascal affirme que «c'est même le nom» que Dieu «se donne dans les Écritures, Deus absconditus» (427), et, lançant les principes de son apologétique, il lie la condition naturelle de l'homme dans le monde, privé de toute relation avec le divin, avec sa compréhension apophatique de la religion chrétienne. Pour Pascal, «ce qui y paraît ne marque ni une exclusion totale, ni une présence manifeste de divinité, mais la présence d'un Dieu qui se cache. Tout porte ce caractère» (449). Pascal nous présente une lecture singulière du christianisme et la rapporte à l'état d'ambiguïté de l'homme dans le monde, «incompréhensible que Dieu soit et incompréhensible qu'il ne soit pas" (809), mettant à la fois en suspension l'indifférence sceptique (Pyrrhoniens et Montaigne) et le dogmatisme théologique (saint Augustin) et onto-théologique (Descartes) qui ssimposèrent comme des modèles traditionnels dıinterprétation de la religion. Sradressant aux athées libertins, et les plaçant dans le même bateau dans lequel les croyants naviguent, Pascal considéra que

Dieu est ou il n'est pas; mais de quel côté pencherons-nous? La raison n’y peut rien déterminer. Il y a un chaos infini qui nous sépare. Il se joue un jeu à l'extrémité de cette distance infinie, où il arrivera croix ou pile. Que gagerez-

Ce n'est pas dans Montaigne mais dans moi que je trouve tout ce que j’y vois (689). 
vous ? Par raison vous ne pouvez faire ni l'un ni l'autre [...] Ne blâmez donc pas de fausseté ceux qui ont pris un choix, car vous n'en savez rien. [...] Oui, mais il faut parier. Cela n'est pas volontaire, vous êtes embarqués (418).

L'argument pascalien évoque une déconstruction du vocabulaire dogmatique du théisme et de l'athéisme, en même temps que renonce à l'appareil onto-théologique de Descartes qui cherchait à certifier l'existence de Dieu à travers de le concept: «la raison n’y peut rien déterminer». Pour Pascal, l'impuissance cognitive qui berce le mouvement de «ceux qui ont pris un choix» de la religion est la même qui accompagne ceux qui sont enclins à la rejeter: «ne cherchons donc point d'assurance et de fermeté; notre raison est toujours déçue par l'inconstance des apparences: rien ne peut fixer le fini entre les deux infinis qui l'enferment et le fuient» (199).

Ce que la fortune critique qualifia de « vision tragique » et qui éloigne Pascal de ses interlocuteurs, Descartes, Montaigne et les disciples de Saint Augustin à Port Royal, à savoir, le rationalisme, le scepticisme et le dogmatisme théologique, fut chirurgicalement décrit dans l>un de ses aphorismes: «nous sommes incapables de ne pas souhaiter la vérité et le bonheur et sommes incapables ni de certitude ni de bonheur». (401). La distance envers lrataraxie pyrrhonienne et le scepticisme de Montaigne se fait jour à travers dıun tempérament religieux incapable «de ne pas souhaiter la vérité et le bonheur» et, en même temps, de se rendre compte quiils sont inaccessibles. Le refus de la lassitude sceptique, qui nıa pas pour principe une recherche de valeurs qui transcendent l`équivocité d`un monde dans lequel «le mal est aisé. Il y en a une infinité», et «le bien presque unique» (526), fait de Pascal lıun des moralistes modernes les plus sophistiqués. Contrairement à beaucoup de ses contemporains, l'observation empirique de la nature et de l'homme apparaît chez Pascal sous la forme d'une lamentation tragique : "prétendent-ils nous avoir bien, de nous dire qu'ils tiennent que notre âme n'est qu'un peu de vent et de fumée, et encore de nous le dire d'un ton de voix fier et content? Est-ce donc une chose à dire gaiement? et n'est-ce pas une chose à dire tristement, au contraire, comme la chose du monde la plus triste? (427).

Les Pensées de Pascal s'inscrivent dans un arc épistémologique qui englobe la tradition sceptique et le rationalisme cartésien et ont, dans la thématisation des éléments du christianisme, le principe d'articulation d'une anthropologie philosophico-religieuse. Dans le fragment 406, nous lisons: «Nous avons une impuissance de prouver, invincible à tout le dogmatisme. 
Nous avons une idée de la vérité invincible à tout le pyrrhonisme». L'impuissance de la raison face à l'infinité des choses qui la dépassent (186), l’humiliation provoquée par la vérité de la connaissance naturelle, la disproportion de l'homme avec la nature et l'inutilité de «ces titres si ordinaires, Des principes des choses, Des principes de la philosophie, et aux semblables aussi fastueux» (199) traduisent une architecture de la nature humaine qui présente comme résultat logique un nihilisme moral. Le mépris de Pascal pour le laxisme moral du sceptique et pour une suspension du jugement sur la vérité qui s'abandonne sans aucune résistance au relativisme des habitus marque son activité de moraliste et penseur religieux. Ce qui sépare Pascal de Montaigne et de la tradition sceptique pyrrhonienne, c'est l'idée de vérité qui ne permet pas au moraliste de se débarrasser des inquiétudes suscitées par l'ambiguïté du monde. Cependant, pour Pascal, le scepticisme pyrrhonien est correct dans la mesure où il révèle l'impuissance du dogmatisme et la fausse sécurité dérivée des puissances trompeuses de la raison. C'est dans ce contexte qu'il faut lire la déclaration de Pascal: «Le pyrrhonisme est le vrai» (691). Dans la critériologie pascalienne, le pyrrhonisme est la vérité parce qu'il décrit philosophiquement l'ambiguité d'un état dans lequel «la nature ne m'offre rien qui ne soit matière de doute et d'inquiétude» (429). C'est à travers cette perception que le scepticisme apparaît comme propédeutique à la pensée religieuse et, par conséquent, dans le contexte des controverses épistémologiques menées par Pascal, «le pyrrhonisme sert à la religion» (658).

\section{Disproportion et expérience mystique}

La compréhension de Pascal de la religion est traversée par un regard qui a appris à contempler l'abîme en silence. Il n’y a rien dans sa pensée qui ne soit traversé par l'état d'ambiguité de l'homme dans le monde. Son renoncement à l'ataraxie pyrrhonienne et à une tranquillité sceptique [qui soutiendrait une posture blasée face à l'équivocité du monde] cédait la place à un approfondissement philosophico-religieux de cette même équivocité. Pour Pascal,

Nous voguons sur un milieu vaste, toujours incertains et flottants, poussés d'un bout vers l'autre; quelque terme où nous pensions nous attacher et nous affermir, il branle, et nous quitte, et si nous le suivons il échappe à nos prises, nous glisse et fuit d'une fuite éternelle; rien ne s'arrête pour nous. C'est l'état qui nous est naturel et toutefois le plus contraire à notre inclination. Nous 
brûlons du désir de trouver une assiette ferme, et une dernière base constante pour y édifier une tour qui s'élève à l'infini mais tout notre fondement craque et la terre s'ouvre jusqu'aux abîmes (199)

Dans l'espace de la nature, les mouvements de l'homme se déplacent à la hâte ou lentement, du vide au rien, comme l'écrivit le moraliste russe qui, dans les profondeurs de l'être souterrain, constata que qu'aucune cause première (principes des choses or principes de la philosophie) ne pourrait avoir lieu (Dostoïevski, 1992). À chaque mouvement de pensée, «un nouvel abîme» (199), et il n'y a pas de vie intérieure, de nature ou même de métaphysique capable d'apaiser les abîmes qui se multiplient à l'infini. En refusant les modèles philosophiques qui prônaient un principe métaphysique pour penser le divin, comme postulé par la causalité aristotélicienne ou par l'intériorité conceptuelle de Descartes, Pascal provoqua une déconstruction avant la lettre de la métaphysique qui offrit une configuration anarchique à son herméneutique du christianisme : «Feu. Dieu d'Abraham, Dieu d'Isaac, Dieu de Jacob, non des philosophes et des savants» (913). Les mots qui ouvrent le Mémorial reflètent le retrait de Pascal d'une expérience philosophique ou religieuse qui se revendique d'une ontologie ou d'un principe métaphysique comme exigence fondamentale de la pensée, qu'il soit nommé comme intériorité, nature ou même Dieu. L'anarchie de la grammaire philosophico-religieuse pascalienne renonce à ces principes en supposant que, tôt ou tard, «tout notre fondement craque et la terre s'ouvre jusqu'aux abîmes».

Analysés à la lumière des critères pascaliens, les concepts d'expérience religieuse et d'expérience mystique acquièrent un sens sans corrélation avec leurs formes traditionnelles, tels qu'ils apparaissent dans l'histoire de la pensée chrétienne. On sait que le dépassement de l'argument cosmologique ou, comme Kant l'a compris, cosmothéologique ${ }^{2}$, et la constitution d'une intériorité moderne ont offert le scénario de l'émergence du vocabulaire mystique aux XVIe et XVIIe siècles ${ }^{3}$. Michel de Certeau montra comment

E. Kant, Critique de la raison pure. Aubier, Paris, 1997, p.553.

De Certeau, M. La faiblesse de croire. Éditions du Seuil, 1987. "le "thème» symbolique de Thérèse ne dit plus la structure d'un objet cosmique, mais celle du sujet; il transpose en une anthropologie l'ancienne cosmologie. Le cosmos (dont le fond est la terre où descende l'influx céleste et d'où s'élève l'âme pour rejoindre l'empyrée) devient microcosme humain: «globe» comme le monde ancien et "cristal" comme la bulle, c'est un monde que chaque sujet constitue à lui seul, dont le centre est "résidence» de Dieu et dont l'environnement est "abîme d'obscurité" [...] La détérioration d'un univers devient pour Thérèse le langage 
la perception de l'infini dans l'homme est devenue le point de départ de la reconstruction d'un ordre dans l'univers et l'élément commun entre une grammaire philosophique cartésienne et le langage spirituel des mystiques. Pour Certeau «l'expérience des mystiques est analogue à celle des plus audacieux parmi leurs contemporains. De son côté, parti 'comme un homme qui marche seul et dans les ténèbres', Descartes découvrira dans le cogito l'innéité actuelle de l'idée de Dieu»(CERTEAU, 1987, p.52). La résistance de Pascal aux puissances trompeuses du sujet cartésien est bien connue et on comprend que, dans la critériologie philosophico-religieuse des Pensées, elle pourrait s'appliquer, peut-être moins incisivement, non pas à l'expérience religieuse des spirituels, mais à ce que $\mathrm{M}$. de Certeau appela le destin prodigieux du mot mystique au XVII siècle» (Ibid, p.52).

Les références au concept d'expérience mystique dans l'ensemble des Pensées sont rares et, cependant, sont assez illustratives. Le nom de sainte Thérèse est mentionné trois fois dans l'ouvrage et dans chacune d'elles nous trouvons ce que nous pourrions appeler, si une telle expression est légitime, un exercice de démystification de l'expérience mystique - qui fut conséquence de la compréhension pascalienne du sens de l'expérience religieuse. Pour Pascal,

Ce qui nous gâte pour comparer ce qui s'est passé autrefois dans l'Église à ce qui s'y voit maintenant est que ordinairement on regarde saint Athanase, sainte Thérèse et les autres, comme couronnés de gloire et d'ans, jugés avant nous comme des dieux. A présent que le temps a éclairci les choses cela paraît ainsi, mais au temps où on le persécutait ce grand saint était un homme qui s'appelait Athanase et sainte Thérèse une fille. Élie était un homme comme nous et sujet aux mêmes passions que nous, dit saint Pierre pour désabuser les chrétiens de cette fausse idée, qui nous fait rejeter l'exemple des saints comme disproportionné à notre état (598).

Le destin prodigieux du mot mystique porta la puissance d'une expérience qui fut, aux côtés du cogito cartésien, fondateur de la modernité (GUERREIRO, 2007). L'excès du langage mystique des spirituels qui percevaient en eux-mêmes une transcendance fondatrice de l'intériorité, établissant ainsi un tournant dans la spiritualité chrétienne, provoqua chez

d'un autre univers, celui-là anthropologique. Le désarroi qui dépouillait l'homme de son monde et, simultanément, des signes objectifs de Dieu, est précisément pour lui le rendezvous de sa renaissance spirituelle. C’est là que le fidèle trouve le signe de Dieu, certitude désormais établie sur une conscience de soi. Il découvre en lui-même ce qui le transcende et ce qui le fonde dans l'existence. P.50-51 
Pascal un jugement qui déplaça ces spirituels du milieu aristocratique dans lequel ils sont traditionnellement placés. L'aristocratie spirituelle des mystiques est, pour Pascal, une erreur de réception, car «ce grand saint était un homme qui s'appelait Athanase et sainte Thérèse une fille». Il y a chez Pascal un exercice de langage qui voulut confronter l'excès de vocabulaire mystique à la modestie d'une philosophie religieuse qui se caractérisa par son effort pour dessiner les contours et les limites d'une «science de l'expérience» (CERTEAU, 1987, p.53). La pensée religieuse de Pascal ne nie donc pas l'expérience mystique tout court, mais plutôt sa dimension d'exceptionnalité, et cherche à la circonscrire aux limites traditionnelles de l'expérience religieuse. En ce sens, on pourrait dire que l'expérience religieuse de la jeune Thérèse ne diffère qualitativement en rien de la modeste conversion d'un paysan de campagne. Les deux expériences sont plongées dans l'équivocité et dans la disproportion absolue de l'homme par rapport à l'infini. La non-participation et la séparation radicale entre la condition de l'homme et ce qui le dépasse, les deux infinis qui ne permettent pas une métaphysique ni une science de l'expérience, soumettent l'expérience philosophique et l'expérience mystique à un état infranchissable d'équivocité et de misère. Dans l'aphorisme 721, nous lisons: «l'ardeur des saints à chercher le vrai était inutile si le probable est sûr. La peur des saints qui avaient toujours suivi le plus sûr. Sainte Thérèse ayant toujours suivi son confesseur».

Un élément déterminant dans la pensée philosophico-religieuse de Pascal fut son rejet de l'intériorité comme espace qualitatif de discernement et de sens de la réalité. Ce point nous rapproche de son jugement sur le cadre philosophique et religieux dans lequel nous retrouvons l'avènement du vocabulaire mystique. La perte du cosmos qui, comme le montre M. de Certeau, définit les cadres mentaux des XVIe et XVIIe siècles et détermine l'existence et l'objet de connaissance d'un homme qui devait «chercher en lui une certitude et une règle», (CERTEAU, 1987, p.48), c'est le principe qui entrelace la découverte d'une intériorité philosophique et le langage mystique des spirituels. Dans le fragment intitulé «philosophes», Pascal considère que

Nous sommes pleins de choses qui nous jettent au-dehors. Notre instinct nous fait sentir qu'il faut chercher notre bonheur hors de nous. Nos passions nous poussent au-dehors, quand même les objets ne s'offriraient pas pour les exciter. Les objets du dehors nous tentent d'eux-mêmes et nous appellent quand même nous n'y pensons pas. Et ainsi les philosophes ont beau dire: rentrez-vous en vous-mêmes, vous y trouverez votre bien; on ne les croit pas 
et ceux qui les croient sont les plus vides et les plus sots (143)

Le contexte philosophique des Pensées est traversé par la philosophie stö̈cienne et le scepticisme pyrrhonien et Pascal cherche à confronter les échos de cette tradition dans le vocabulaire philosophique et religieux de ses contemporains, notamment Descartes et Montaigne. Pour Pascal, «ce que les stö̈ques proposent est si difficile et si vain»(144), parce qu'ils disent, «rentrez au-dedans de vous-même, c'est là où vous trouverez votre repos. Et cela n'est pas vrai» (407). L'ascétisme stoïcien contemple l'extériorité du monde à travers une expérience de soi qui présuppose un «moi» qui se présente, selon l'expression d'Épictète, à travers l'image d'une «citadelle intérieure» (BENSUSSAN, 2019, p.19). Ce principe d'intériorité marque le stoïcisme et il ne serait pas faux d'associer l'intériorité stö̈cienne aux éléments fondateurs du récit mystique. En interprétant l'expérience mystique à travers les catégories traditionnelles de l'expérience religieuse du christianisme et en plaçant l'expérience de sainte Thérèse dans la vie prosaïque de la foi chrétienne, Pascal signale que l'intériorité, comprise comme le centre de la doctrine stö̈cienne du bien, ne saurait être interprétée comme le principe ou le lieu de discernement de la vérité et de la réalité. Ce "je-là", comme Gérard Bensussan remarque à propôs d'Epictète, "est une fiction" (ibid). Le moi pour Pascal se montre à travers ses «qualités empruntées» et ne porte aucune substance qui puisse être assimilée à l'abstraction de l'image stoïque 4 .

Il ne serait pas faux de considérer qu'il y chez Pascal une résistance philosophique à une pratique spirituelle qui présuppose la notion stö̈cienne d'un «je assuré de soi» (ibid) et, ce qui en est une conséquence, un exercice spirituel de mystification d'une intériorité qui pourrait elle-même donner sens et substance à l'extériorité du monde. L'idée d'intériorité, comme la notion

4 Qu'est-ce- que le moi? Un homme qui se met à la fenêtre pour voir les passants; si je passe par là, puis-je dire qu'il s'est mis là pour me voir? Non; car il ne pense pas à moi en particulier; mais celui qui aime quelqu'un à cause de sa beauté, l'aime-t-il? Non: car la petite vérole, qui tuera la beauté sans tuer la personne, fera qu'il ne l'aimera plus. Et si on m'aime pour mon jugement, pour ma mémoire, m'aime-t-on? moi? Non, car je puis perdre ces qualités sans me perdre moi-même. Où est donc ce moi, s'il n'est ni dans le corps, ni dans l'âme? et comment aimer le corps ou l'âme, sinon pour ces qualités, qui ne sont point ce qui fait le moi, puisqu'elles sont périssables? Car aimerait-on la substance de l'âme d'une personne, abstraitement, et quelques qualités qui y fussent? Cela ne se peut, et serait injuste. On n'aime donc jamais personne, mais seulement des qualités. Qu'on ne se moque donc plus de ceux qui se font honorer pour des charges et des offices, car on n'aime personne que pour des qualités empruntées (688). 
stoïcienne de la nature, si l'on considère «où nous mènent les connaissances naturelles» (199), apparaît chez Pascal comme des réalités fictives qui rendent possible une pratique spirituelle naïve : «ceux qui les croient sont les plus vides et les plus sots» (143). Conscient de son état de disproportion par rapport à la nature et, par conséquent, privé de toute ontologie, Pascal reconnut dans le moi stoïcien une abstraction et dans les exercices spirituels de l'ascétisme antique ou chrétien, s'ils partagent la même idée de l'intériorité, une activité prosaïque qui pourrait être classée comme un divertissement.

La résistance de Pascal au stoïcisme aide à comprendre son jugement sur l'expérience mystique et éclaire une critériologie philosophico-religieuse qui suppose la condition d'un homme «incapable de voir le néant d'où il est tiré et l'infini où il est englouti» (199). Les espaces traditionnels de médiation [et de méditation], tels qu'ils apparaissent dans le stoïcisme et dans les pratiques spirituelles de caractère mystique et ascétique, sont rejetés au nom d'une herméneutique du christianisme qui suppose la présence et l'absence d'un dieu caché. La religion chrétienne assume, selon Pascal, l'équivocité du monde, et celui-ci est le sens qui fait des Pensées une apologétique du christianisme. Pour le philosophe de Port Royal, «Dieu étant ainsi caché toute religion qui ne dit pas que Dieu est caché n'est pas véritable, et toute religion qui n'en rend pas la raison n'est pas instruisante. La nôtre fait tout cela. Vere tu es deus absconditus» (242). L'expression deus absconditus, prise par Pascal de la tradition prophétique du livre d'Isaïe, traduit le noyau du monothéisme juif et suppose une profanation complète de l'idée de nature ainsi que la transcendance absolue de la divinité. Cette appropriation donna forme à une compréhension de la religion et de l'expérience religieuse qui présuppose, comme on le trouve dans le judaïsme biblique, le caractère non mystique de l'expérience avec le divin et la séparation radicale entre Dieu et l'homme ${ }^{5}$

\section{Religion, contingence et altérité}

La pensée de Pascal se dessine dans une architecture philosophicoreligieuse dénuée de principes. Résistant à tout ordre qui a pris une vie mystique avec des racines néoplatoniciennes, une philosophie et théologie de la nature, ou même une ontothéologie qui soutenait l'existence de ce qu'il nomma lui-même le dieu des philosophes, Pascal donna naissance à ce

Cf. GUTTMANN Julius, Histoire des philosophies juives de l'époque biblique à Franz Rosenzweig, Paris, Gallimard, 1994. Pour Pascal, «les vrais juifs et les vrais chrétiens n’ont qu’une même religion» (453) 
que l'on pourrait appeler, à la suite des intuitions de Jean Luc-Nancy, d'une configuration anarchique du christianisme. Pour Nancy,

Ce n'est pas un hasard si en plein essor de la rationalité moderne, Pascal éprouve si violemment, dans son « Mémorial », la nécessité de disjoindre sans réserve le Dieu «des philosophes et des savants" du «DIEU d'Abraham, d'Isaac et de Jacob, DIEU de Jésus-Christ». Cette disjonction et la contrariété ou la contradiction qui en résulte sont logées au cœur de l'athéisme, très exactement à l'endroit où le principe même du principe s'effondre de lui-même et, dans cet effondrement, signale la possibilité, voire l'exigence et l'appel d'une tout autre configuration anarchique (NANCY, 2005, p.38-39).

Le principe même du principe, pour reprendre l'expression de Nancy, qui s'effondre dans la pensée pascalienne, atteste l'état d'équivocité de l'homme et de ce qui le dépasse en admettant l'inexistence de toute expérience d'union, voire de fusion avec «l'infini». La conscience de rupture avec la nature et la conscience des états presque fictifs du moi, ses «qualités empruntées», ouvre un abîme dans l'existence qui, dépourvu de toute causalité et identité, assume un rapport d'altérité absolue à l'extériorité et avec soi-même. C'est dans ce prisme anti-métaphysique que nous devons lire la lamentation pascalienne: «le silence éternel de ces espaces infinis m'effraie» (201). Pascal énonce une condition d'équivocité qui éloigne son christianisme des formulations métaphysiques traditionnelles, comme on le trouve, par exemple, dans ce que Jean-Luc Nancy appela «stoïcisme-chrétiene», qui trouva au nom de Dieu rien de plus qu'une manière de nommer «le principe de une totalité présupposée, fondée en unité et en nécessité (NANCY, 2005, p. 35). La perte de l'unité cosmothéologique du monde et le refus pascalien d'une vie intérieure [compris ici comme une expérience philosophique ou mystique capable de façonner, ascétiquement ou conceptuellement, une autre expérience d'unité d'existence], offrîrent ainsi le scénario pour la formulation pascalienne d'un christianisme tragique. Dans l'aphorisme (429), on lit:

Voilà ce que je vois et ce qui me trouble. Je regarde de toutes parts, et je ne vois partout qu'obscurité. La nature ne m'offre rien qui ne soit matière de doute et d'inquiétude. Si je n’y voyais rien qui marquât une Divinité, je me déterminerais à la négative; si je voyais partout les marques d'un Créateur, je reposerais en paix dans la foi. Mais, voyant trop pour nier et trop peu pour m’assurer, je suis dans un état à plaindre, et où j'ai souhaité cent fois que, si un Dieu la soutient, elle le marquât sans équivoque; et que, si les marques 
qu'elle en donne sont trompeuses, elle les supprimât tout à fait; qu'elle dît tout ou rien, afin que je visse quel parti je dois suivre. Au lieu qu'en l'état où je suis, ignorant ce que je suis et ce que je dois faire, je ne connais ni ma condition, ni mon devoir. Mon cœur tend tout entier à connaître où est le vrai bien, pour le suivre; rien ne me serait trop cher pour l'éternité. Je porte envie à ceux que je vois dans la foi vivre avec tant de négligence, et qui usent si mal d'un don duquel il me semble que je ferais un usage si différent

Pascal fut le premier penseur moderne à proposer, à travers son opposition entre le dieu des philosophes et le dieu d'Abraham, une herméneutique du christianisme en tant que déconstruction des fondements de la métaphysique. Partant d'un scepticisme qui renonça aux puissances trompeuses de la raison, ses excès de subjectivité, Pascal renonce aussi à une mystification de la nature ou de la vie intérieure provenant d'une spiritualité naïve. De ce point de vue, Pascal donna naissance à un concept d'expérience religieuse qui est contraire à tout principe (ontothéologique, cosmothéologique ou mystique) et offrit un nouveau sens, à la lumière de la vie moderne, au concept de foi que l'on retrouve dans la tradition juive et chrétienne. L'observation de Pascal sur le fait qu'il n'y a rien dans la nature qui ne soit «matière de doute et d'inquiétude», l'ignorance complète de soi-même et de sa vie intérieure, présenta le point de départ pascalien, qui fut son propre nihilisme, pour une herméneutique du christianisme. Le gouffre qui sépara la pensée de Pascal de la métaphysique, ou, comme le dit Jean-Luc Nancy, de «toute l'histoire de 'Dieu' - du Dieu de l'Occident'» (NANCY, 2005, p.35), explique aussi sa distance et jugement par rapport à une herméneutique dogmatique du christianisme. Les preuves de l'existence de Dieu que l'on trouve dans des œuvres telles que De Libero arbitrio et De vera religione, de Saint Augustin, qui constituèrent une tradition dogmatique qui s'étendirent au Proslogion de Saint Anselme et traversèrent l'imaginaire religieux de Port Royal, ont été mises en suspension par le doute sceptique de Pascal ${ }^{6}$.

L'apologétique du christianisme présente dans les Pensées doit être interprétée avant la lettre comme une déconstruction de la métaphysique qui, en revenant à la grammaire du monothéisme juif, formula une expérience du christianisme privé de toute articulation dogmatique. Pour Pascal, il n’y

6 «S'il y a un Dieu il est infiniment incompréhensible, puisque n'ayant ni parties ni bornes, il n'a nul rapport à nous. Nous sommes donc incapables de connaître ni ce qu'il est, ni s'il est. Cela étant qui osera entreprendre de résoudre cette question? ce n'est pas nous qui n'avons aucun rapport à lui»(418). 
a pas de certitudes positives dans le discours religieux et, de même, un degré radical d'incertitude détermine les limites du discours rationnel. En ce sens, n'était la demande de vérité provenant du tempérament religieux de Pascal, l'ataraxie pyrrhonienne, accompagnée de divertissements qui offrent de nombreuses expériences d'enrichissement de l'existence, serait un chemin philosophique possible et la religion une simple excentricité. Cependant, chez Pascal, le «cœur tend tout entier à connaître où est le vrai bien» (429), et celui-ci est le sentiment qui provoque son mépris pour le nihilisme indifférent des Pyrrhoniens. Ainsi, en rejetant le dogmatisme des disciples de saint Augustin, Pascal élucide une expérience religieuse qui s'ancre à l'insurmontable contingence de l'homme et atteste l'extériorité et l'altérité radicale d'une réalité que lui échappe.

S'il ne fallait rien faire que pour le certain on ne devrait rien faire pour la religion, car elle n'est pas certaine. Mais combien de choses fait-on pour l'incertain, les voyages sur mer, les batailles. Je dis donc qu'il ne faudrait rien faire du tout, car rien n'est certain. Et qu'il y a plus de certitude à la religion que non pas que nous voyions le jour de demain. Car il n'est pas certain que nous voyions demain, mais il est certainement possible que nous ne le voyions pas. On n'en peut pas dire autant de la religion. Il n'est pas certain qu'elle soit mais qui osera dire qu'il est certainement possible qu'elle ne soit pas. Or quand on travaille pour demain et pour l'incertain on agit avec raison, car on doit travailler pour l'incertain par la règle des partis qui est démontrée. Saint Augustin a vu qu'on travaille pour l'incertain sur mer, en bataille, etc. - mais il n'a pas vu la règle des partis qui démontre qu'on le doit. Montaigne a vu qu'on s'offense d'un esprit boiteux et que la coutume peut tout, mais il n'a pas vu la raison de cet effet (577).

L'argument de Pascal censure Augustin le fait qu'il n'a pas porté jusqu'à ses ultimes conséquences l'incertitude dans l'existence humaine et d'avoir nourri avec cela un dogmatisme qui donne à l'expérience religieuse les certitudes positives de la raison et de la tradition ${ }^{7}$. Contrairement à la fortune critique augustinienne qui a trouvé dans le récit dogmatique du christianisme un moyen de certifier la religion, Pascal considère que de la même manière que dans les batailles et les voyages l'homme se trouve face à l'incertain, la religion, à son tour, n'est pas certaine non plus. Ignorant l'équivocité radicale de l'état qui nous est naturelle, la philosophie ainsi

Pour cela, nous renvoyons à la thèse de Lucien Goldmann qui est ici à la base de notre argumentation (GOLDMANN, 1959, p.321). 
que la religion sont confrontées au risque de «édifier une tour qui s'élève à l'infini» (199). Pour Pascal, c'est le même mouvement de l'esprit qui structure les certitudes de la religion qui offre les fondements dogmatiques d'un athéisme qui cherche à nier la possibilité même de croyance. Comme il le soutient, «qui osera dire quil est certainement possible qu>elle - la religion - ne soit pas». La sophistication de lyargument de Pascal veut démontrer la similitude entre deux attitudes dogmatiques qui sıimaginent capables dıun mouvement drappropriation d`une réalité qui les dépasse. Dans le fragment (157) il considère : «athéisme marque de force d'esprit, mais jusqu'à un certain degré seulement». Car, sur le prisme pascalien, il n'est pas tout à fait certain que l'extériorité du monde nous présente le rien, au lieu de quelque chose, ni parfaitement clair que «l'âme soit matérielle» (161). Il n'est pas du tout inintéressant de se rendre compte que l'argument de Pascal se fait connaître dans une philosophie de la religion qui cherche à élaborer une suspension du dogmatisme naï, souvent vulgaire, de l'athéisme moderne. Des concepts tels que «absentéisme», utilisé par Jean-Luc Nancy pour s'opposer au concept d'athéisme (NANCY, p. 32), ou «anathéisme», proposé par Richard Kearney, afin de surmonter l'opposition entre le théisme dogmatique et l'athéisme (KEARNEY, 2010), se rapprochent des intuitions de Pascal et de son herméneutique du christianisme.

Le retrait du christianisme de la métaphysique opéré par Pascal provoqua un désordre qui rendit inopérant les «exercices spirituels» drarticulation du sens provenant d`une expérience philosophique et/ou mystique qui présupposent, dans leurs spécificités, un contact immédiat avec le divin (LAUX, 2005, p.77). Les affinités électives de Pascal avec le judaïsme sont, en ce sens, plus quévidentes, et ladage d`Isaïe, Deus absconditus, qui constitua aussi le principe d'une intelligence philosophique juive, Franz Rosenzweig et Emmanuel Levinas, par exemple, ont traduit une philosophie de la religion qui renforça la disproportion qui règne entre l'homme et le divin. Ainsi, la profanation du monde imposée par le monothéisme et par la transcendance absolue du Dieu juif, qui conduisit Schelling à percevoir la symbiose entre le monothéisme et l'athéisme (NANCY, p. 27), doit être interprété comme toile de fond de l'argumentation pascalienne et comme partie de sa grammaire religieuse. Pour Pascal, il n'y a rien dans la physicalité propre de la nature et à l'intérieur de l'homme qui permet d'envisager une expérience d'unité et / ou de participation de l'homme à la nature divine. Le désordre pascalien, principe fondamental de l'état naturel de l'homme, suppose une physis sans ontologie et une psychologie 
déracinée de toute architecture métaphysique. En provoquant un affolement des concepts en vue de «destituer la métaphysique», Pascal renonce aussi à l'idée d'infini comme concept présupposé pour la nomination de Dieu. Comme écrit Jean-Luc Marion, pour Pascal «l'infini devint, de nom divin et détermination privilégiée de Dieu, l'indice multiplié sans fin de l'incommensurabilité des éléments éclatés de ce qui ne peut plus se nommer un cosmos; de concept, il se trouve ravalé au rang d'une métaphore utilisée à défaire d'autres concepts» (MARION, 2004, p.325).

La méfiance de Pascal à l'égard de la métaphysique consacra dans la modernité et aussi au sein du christianisme l'opposition classique entre Jérusalem et Athènes. L'iconoclasme philosophique de sa pensée pointa un arsenal critique vers: i) une philosophie et / ou mystique qui présuppose l'union de l'âme avec le divin selon les modèles de la tradition platonicienne et néoplatonicienne, ii) une philosophie de la nature qui revendique une proportion et / ou une identité entre l'homme et la nature, et, enfin, iii) une spiritualité ou mystique du christianisme qui propose une analogie entre l'homme et Dieu et revendique la possibilité d'une compréhension ou cognitio du divin. Le christianisme de Pascal, en ce sens, s'est organisée en dehors de la grammaire de l'hellénisme, fondement et lieu de constitution du dieu de la philosophie, se rapprochant ainsi d'une grammaire du judaïsme qui donna voix à la contingence absolue de l'homme et à l'altérité radicale du vrai et du bien: vere tu es deus absconditus.

Les Pensées de Blaise Pascal doivent être lues, contre la naïveté des pratiques spirituelles contemporaines, comme un bréviaire moderne.

\section{Bibliographie}

BENSUSSAN, G. Être heureux? Ce qui dépend de nous et ce qui n’en dépend pas.

Éditions Mimesis/Philosophie et Societé. N.11. Collection dirigée par Yves Charles Zarka. Paris, 2019.

DE CERTEAU, M. La faiblesse de croire. Éditions du Seuil, 1987

DOSTOÏEVSKI, F. Les Carnets du sous-sol. (Traduction d'André Markowicz), Actes Sud, 1992.

GUERREIRO, E. Teologia negativa e Theodor Adorno. A secularização da mística na arte moderna. Tese de Doutorado. http://www.posciencialit.letras.ufrj.br/images/ Posciencialit/td/2007/21-eduardoguerreiro_teologianegativa.pdf. Accès le 28 juin 2020.

GOLDMANN, L. Le Dieu Chaché. Étude sur la vision tragique dans les Pensées de Pascal et dans le théâtre de Racine. Éditions Gallimard, 1959. 
KEARNEY, R. Anatheism: Returning to God after God. New York: Columbia University Press, 2010.

KOYRÉ, Alexandre. From the closed world to the infinite universe. Baltimore, Johns Hopkins Press, 1957.

LAUX, Henri. Qu'est-ce que la mystique ? In. Capelle, Philippe (ed). Expérience Philosophique et Expérience mystique, Paris, 2004.

MARION, Jean-Luc. Sur le prisme métaphysique de Descartes. Constitution et limites de l'onto-théo-logie dans la pensée cartésienne. Paris: Press Universitaires de France, 2004

MARTINS, Maria Manuela Brito. A prova da existência de Deus em Santo Agostinho (De Libero Arbitrio) e em Santo Anselmo (Proslogion). https://repositorio.ul.pt/ bitstream/10451/24169/1/Philosophica\%2034_5_MManuelaBritoMartins.pdf

NANCY, J L. La Déclosion (Déconstruction du christianisme, 1). Paris : Galilée 2005,

PASCAL, B. Préface sur le traité du vide. In. Pensées, fragments et lettres de Blaise Pascal. Tome premier. Paris, Andrieux, Éditeur, 1844.

PASCAL, B. Pensées sur la religion et sur quelques autres sujets. Paris, Delmas, 1952. Disponible en : https://www.ub.uni-freiburg.de/fileadmin/ub/referate/04/pascal/pensees. pdf. Accès le 28 juin 2020.

PONDÉ, L F. O homem insuficiente. Coleção Ensaios de Cultura. São Paulo: Edusp, 2001.

Submetido em: 18-12-2020

Aceito em: 1-6-2021 